\title{
Coexistence of site- and bond-centered electron localization in the high-pressure phase of $\mathrm{LuFe}_{2} \mathrm{O}_{4}$
}

\author{
G. R. Hearne, ${ }^{1,{ }^{*}}$ E. Carleschi, ${ }^{1}$ W. N. Sibanda, ${ }^{1}$ P. Musyimi, ${ }^{1}$ G. Diguet,,${ }^{1,2}$ Yu. B. Kudasov, ${ }^{3,4}$ \\ D. A. Maslov, ${ }^{3,4}$ and A. S. Korshunov 3,4 \\ ${ }^{1}$ Department of Physics, University of Johannesburg, P.O. Box 524, Auckland Park, 2006, Johannesburg, South Africa \\ ${ }^{2}$ Institut Néel, Centre national de la recherche scientifique (CNRS) and Université Joseph Fourier, 5 rue des Martyrs BP 166, \\ 38042 Grenoble Cedex 9, France \\ ${ }^{3}$ Sarov Physics and Technology Institute, National Research Nuclear University (Moscow Engineering Physics Institute), 6 Dukhov Street, \\ Sarov, Nizhni Novgorod Region, 607186 Russia \\ ${ }^{4}$ Russian Federal Nuclear Center-All-Russian Scientific Research Institute of Experimental Physics (RFNC-VNIIEF), 37 Myra Prospekt, \\ Sarov, Nizhni Novgorod Region, 607188 Russia
}

(Received 4 January 2016; revised manuscript received 3 March 2016; published 28 March 2016)

\begin{abstract}
Magnetic-electronic hyperfine interaction parameters of spectral components are obtained from in situ ${ }^{57} \mathrm{Fe}$ Mössbauer spectroscopy pressure studies of the mixed-valence $\mathrm{LuFe}_{2} \mathrm{O}_{4}$ multiferroic, up to $\sim 30 \mathrm{GPa}$ and on recovered high-pressure phase samples. Temperature-dependent Mössbauer spectra of the low-pressure phase show that $\mathrm{Fe}^{2+}$ and $\mathrm{Fe}^{3+}$ sites are discernible, consistent with known site-centered charge order in the triangular (frustrated) Fe sublattice network. Magnetic spectra of the high-pressure phase, stabilized in a rectangular $\mathrm{Fe}$ sublattice network at $P>8 \mathrm{GPa}$, exhibit fingerprints of iron in an intermediate valence state only. Temperature-dependent resistivity pressure studies evidence thermally activated small polaron motion in the high-pressure phase. These experimental signatures, complemented by $a b$ initio calculations of electronic structure, are considered evidence of asymmetric dimer formation $\mathrm{Fe}^{(2+\Delta+)} \Leftrightarrow \mathrm{Fe}^{(3-\Delta)+}$, where the minority-spin electron deconfinement coefficient is $\Delta=0.3-0.4$. Bragg satellites discerned in electron diffraction patterns of the metastable high-pressure phase possibly stem from this admixture of site- and bond-centered localization (intermediate-state charge order) in a magnetic background. This breaks inversion symmetry and potentially renders $\mathrm{LuFe}_{2} \mathrm{O}_{4}$ in its high-pressure phase as a new charge order instigated (electronic) ferroelectric.
\end{abstract}

DOI: 10.1103/PhysRevB.93.105101

\section{INTRODUCTION}

Charge order (CO) has often been considered as a site-centered superstructure involving transition metal (TM) sites with different discrete valences. Such TM-centered CO is pictured as mobile carriers confined to a TM sublattice (e.g., $t_{2 g}$ minority-spin electron at $\mathrm{Fe}^{2+}$ ) [1]. Examples are alternation of $\mathrm{Fe}^{2+} / \mathrm{Fe}^{3+}$ in $\mathrm{Fe}_{2} \mathrm{OBO}_{3}$ [2], or less definitively $\mathrm{Mn}^{3+} / \mathrm{Mn}^{4+}$ in $\mathrm{La}_{0.5} \mathrm{Ca}_{0.5} \mathrm{MnO}_{3}$ [3,4]. The latter is less definitive because calculations show other energetically favorable $\mathrm{CO}$ states occur, notably (i) bond-centered $\mathrm{CO}$ and (ii) so-called intermediate-state $\mathrm{CO}$, see Refs. [3,5] and the review [6]. In case (i), in TM oxides, oxygen ions on TM-TM bonds (e.g., perovskite) facilitate electron transfer processes, and charge carriers are confined evenly over a TM-TM symmetric dimer [6-8]. In intermediate-state CO, case (ii), an admixture of site- and bond-centered configurations is energetically favored [3,6]. Carrier delocalization involves preferential confinement to a neighboring TM atom and formation of electronically asymmetric TM-TM dimers.

These alternatives to site-centered $\mathrm{CO}$ have generated considerable excitement in: parent or precursor phases of high- $T_{\mathrm{C}}$ cuprates $[9,10]$, colossal magnetoresistance doped manganites [11-13], and so-called type-I multiferroics. The latter include manganites $\operatorname{Pr}_{1-\mathrm{x}} \mathrm{Ca}_{\mathrm{x}} \mathrm{MnO}_{3}$ [13], the famous case of magnetite $\left(\mathrm{Fe}_{3} \mathrm{O}_{4}\right)$ [6], and the spin/charge frustrated

\footnotetext{
*Author to whom all correspondence should be addressed: grhearne@uj.ac.za
}

case $\mathrm{LuFe}_{2} \mathrm{O}_{4}$ [14]. This has spawned a hot topical area in multiferroics where $\mathrm{CO}$ is predicted to render electronically driven ferroelectricity $[6,15]$. Intermediate-state $\mathrm{CO}$ yields requisite inversion symmetry breaking for electronically driven ferroelectric polarization $[3,6]$.

Our focus is $\mathrm{CO}$ in the pressure-temperature phase diagram of $\mathrm{LuFe}_{2} \mathrm{O}_{4}$. This has been intensely scrutinized in the low-pressure (LP) phase, and claims for CO-instigated ferroelectricity at ambient pressure are mired in controversy; see the review [16] and Ref. [17]. Rouquette et al. [18] showed that a high-pressure (HP) polymorph stabilizes above $\sim 8 \mathrm{GPa}$ (hereafter referred to as $\mathrm{LuFe}_{2} \mathrm{O}_{4}$-HP) and is locked-in upon decompression to ambient conditions. It is purported to involve $\mathrm{CO}$, based on: Bragg satellites seen in electron diffraction studies of recovered specimens, resistivity-pressure behavior at room temperature, and detailed structural comparisons of LP and HP polymorphs [18,19]. Damay et al. [19] focused on more detailed crystal-chemical, structural, and magnetic elucidations. Synchrotron $\mathrm{x}$-ray data analysis revealed an LP monoclinic $C 2 / m$ transformation to a monoclinic $P m$, distorted rock-salt type, HP polymorph. Frustration in the LP phase is relieved as the original triangular Fe network changes to rectangular; see Figs. 1(a) and 1(b) [20]. Electron diffraction micrographs of recovered $\mathrm{LuFe}_{2} \mathrm{O}_{4}-\mathrm{HP}$ showed weak satellites with respect to the $\mathrm{Pm}$ parent cell. These are indicative of either charge modulation as proposed earlier by Rouquette et al. [18], or a structural modulation involving the misfit-related structure of the monoclinic HP phase. Recovered $\mathrm{LuFe}_{2} \mathrm{O}_{4}-\mathrm{HP}, \sim 9 \%$ denser than the LP phase, exhibited antiferromagnetic ordering up to $T_{\mathrm{N}} \sim 380 \mathrm{~K}$. 

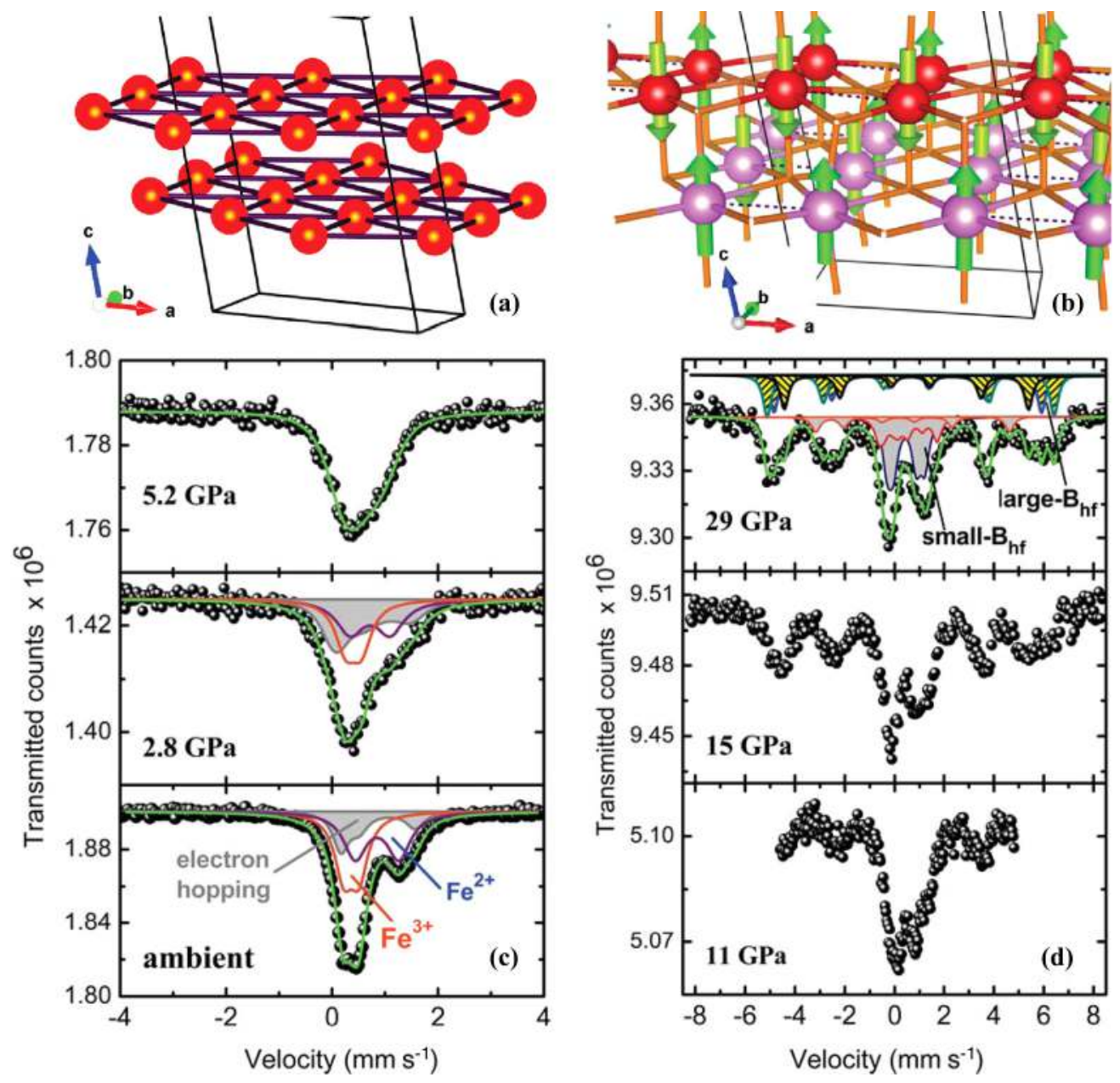

FIG. 1. Bilayers at $300 \mathrm{~K}$ involving (a) triangular networked Fe in the LP phase and (b) rectangular networked Fe in the HP structure where oxygen atoms (not shown) are at vertices of connecting bonds. Vectors on Fe show magnetic moments, and dashed lines represent shortest Fe-Fe distances. ${ }^{57} \mathrm{Fe}$ Mössbauer spectra at $300 \mathrm{~K}$ of (c) paramagnetic LP phase and (d) magnetic HP phase. Solid lines through data points are overall fits to spectra. Deconvolution into subcomponents representing various charge states is discussed in the text.

The nature of any charge segregation (CO) in $\mathrm{LuFe}_{2} \mathrm{O}_{4}-\mathrm{HP}$ is deemed worthy of deeper scrutiny, to potentially unveil a new HP stabilized type-I multiferroic [6]. To this end, our temperature-dependent ${ }^{57} \mathrm{Fe}$ Mössbauer-effect spectroscopy (MS) pressure studies and electronic structure calculations, complemented by resistivity-pressure studies, enable direct probing of the Fe magnetic-electronic state.

\section{EXPERIMENTAL METHODS AND RESULTS}

Experimental methodologies are similar to our previous ${ }^{57} \mathrm{Fe}$ MS and resistivity investigations in diamond anvil cells (DACs) of the pressure response of site-centered $\mathrm{CO}$ in $\mathrm{Fe}_{2} \mathrm{OBO}_{3}$ [21,22]. The highly stoichiometric $\mathrm{LuFe}_{2} \mathrm{O}_{4}$ used here is from previous studies involving an extensive characterization at ambient pressure [23,24]. The recovered HP phase sample from $\mathrm{LuFe}_{2} \mathrm{O}_{4}$, taken to $12 \mathrm{GPa}$ in previous neutron diffraction studies [19], was subjected to ${ }^{57} \mathrm{Fe}$ MS investigations as well.

The Fe magnetic-electronic state is deduced from fitted hyperfine interaction parameters of deconvoluted subcomponents of complex spectral profiles [25]. The most reliably determined parameters are the isomer shift IS (subspectrum centroid) quoted relative to the $\alpha$-Fe metal standard, the quadrupole doublet-splitting $(Q S)$, and the internal magnetic field $B_{h f}$ (sextet hyperfine structure) [26,27]. Further details on experimental methodologies and analyses are provided in the Supplemental Material [28].

The Mössbauer spectral pressure response of LP and HP phases at $300 \mathrm{~K}$ are radically different; see Figs. 1(c) and 1(d), respectively. Paramagnetic spectra of the LP phase are initially a superposition of equally abundant $\mathrm{Fe}^{2+}$ and $\mathrm{Fe}^{3+}$ doublets representing site-centered CO. There is also an electron-hopping component, $\mathrm{Fe}^{2+} \Leftrightarrow \mathrm{Fe}^{3+}$, initiated at $\mathrm{CO}$ domain boundaries at ambient pressure [28,29] (Table S1), the abundance of which increases upon compression; see Fig. 1(c). At $\sim 5.2 \mathrm{GPa}$, beyond the HP-phase onset, the pronounced shoulder (at $\sim 1.5 \mathrm{~mm} \mathrm{~s}^{-1}$ ), signifying discrete valences, merges into the total spectral envelope. This spectrum is fitted with a single electron-hopping component, already hinting at the disruption of site-centered $\mathrm{CO}$ in the HP majority phase.

Our main interest is the spectral attributes of $\mathrm{LuFe}_{2} \mathrm{O}_{4}-\mathrm{HP}$ fully stabilized at $P>8 \mathrm{GPa}$; see Fig. 1(d). The magnetic signature resonances emerge at $\sim 11 \mathrm{GPa}$ in wings of the 

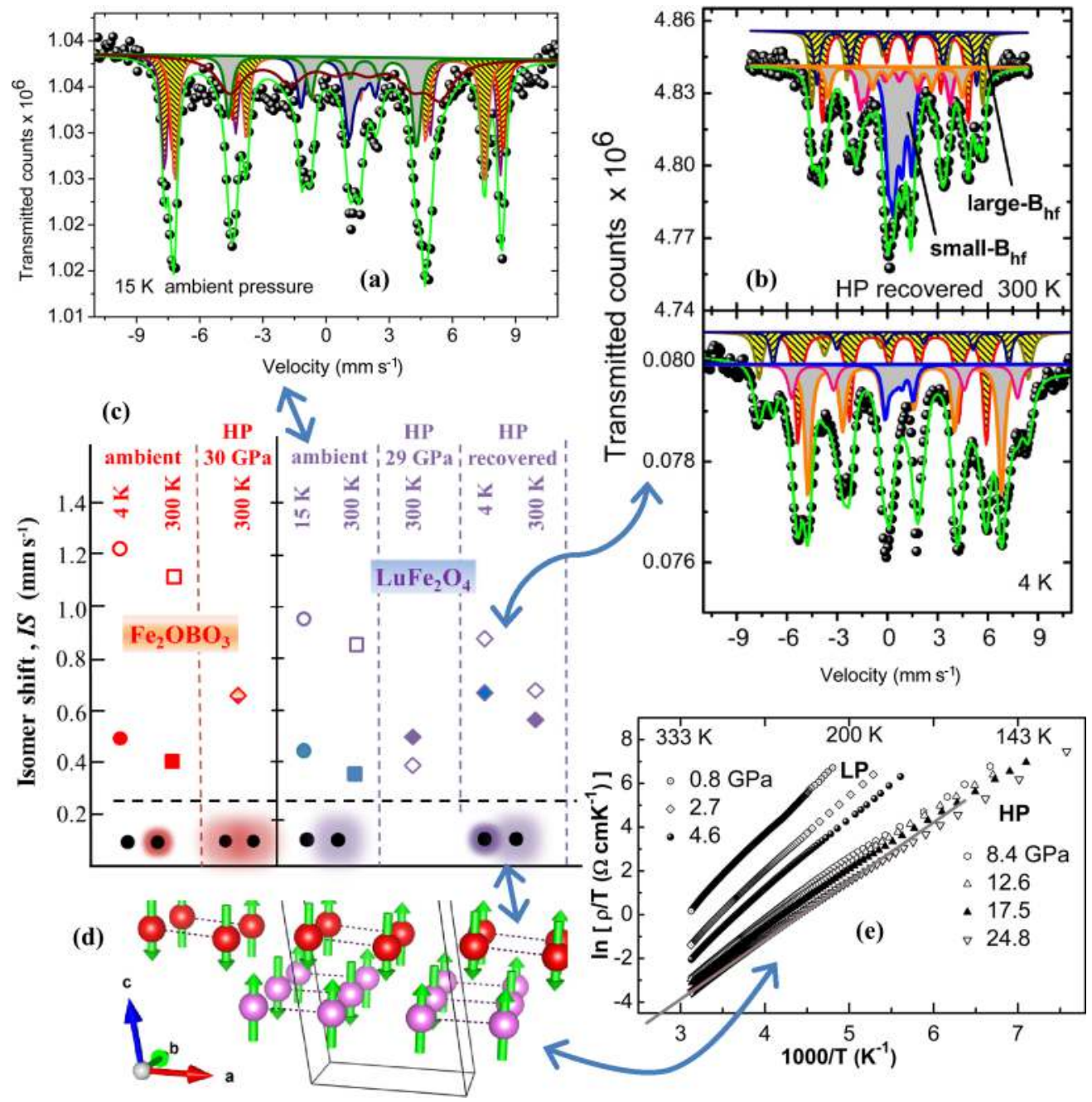

FIG. 2. ${ }^{57} \mathrm{Fe}$ Mössbauer spectra of (a) magnetic LP phase at $15 \mathrm{~K}$ and (b) recovered HP phase. Subcomponents representing Fe ${ }^{(3-\Delta)+}$ and $\mathrm{Fe}^{(2+\Delta)+}$ sites are discussed in the text. Solid lines through data points represent overall fit to a spectrum. Spectrum at $4 \mathrm{~K}$ was measured with a synchrotron Mössbauer source. (c) Valence separation representation, by comparison of isomer shifts (relative to $\alpha$-Fe metal standard) of spectral subcomponents of $\mathrm{LuFe}_{2} \mathrm{O}_{4}$ and $\mathrm{Fe}_{2} \mathrm{OBO}_{3}$ reference. Bottom cartoon depicts minority-spin electron distribution/deconfinement at neighboring Fe-Fe sites. (d) Dimers forming Zener polarons along chains of ferromagnetic atomic spins in the $a$ direction. (e) Plot of $\ln (\rho / T)$ versus inverse temperature. Solid gray line is a linear fit to HP data at $\sim 25 \mathrm{GPa}$ in the range $180-320 \mathrm{~K}$, representing thermally activated small polarons.

spectrum at $300 \mathrm{~K}$ and become more conspicuous in spectra of $\mathrm{LuFe}_{2} \mathrm{O}_{4}$ - $\mathrm{HP}$ at $P>11 \mathrm{GPa}$ on an extended velocity scale, as seen in Fig. 1(d). There is appreciable intensity and asymmetry in the central region near zero velocity. Relatively broad spectral line shapes indicate a multiplicity of $\mathrm{Fe}$ sites.

The magnetic spectrum of $\mathrm{LuFe}_{2} \mathrm{O}_{4}$-HP is first compared with that of the LP phase at ambient pressure, depicted in Fig. 2(a), measured well below the ferrimagnetic ordering onset $T_{\mathrm{M}} \sim 240 \mathrm{~K}$. In the LP phase, three large- $\mathrm{B}_{\mathrm{hf}}$ sextets (46-50 T, hatched yellow shading) with similar IS values $\left(0.45 \mathrm{~mm} \mathrm{~s}^{-1}\right)$ represent $\mathrm{Fe}^{3+}$ sites. Three comparatively small-B $\mathrm{Bf}_{\mathrm{hf}}$ sextets $(7-31 \mathrm{~T}$, shaded gray) with similar IS values $\left(0.95 \mathrm{~mm} \mathrm{~s}^{-1}\right)$ represent $\mathrm{Fe}^{2+}[27,30]$, including an additional diffuse "spin-frustrated" $\mathrm{Fe}^{2+}$ component $[28,31]$ (Table S2). This is guided by previous studies [32-35] and compatibility with the known ferrimagnetic structure and
$\mathrm{Fe}^{2+} / \mathrm{Fe}^{3+}$ superstructure (site-centered $\mathrm{CO}$ ) occurring in each sheet of a bilayer [35-37].

The relatively complex magnetic spectra of both $\mathrm{LuFe}_{2} \mathrm{O}_{4}$ $\mathrm{HP}$ and its recovered metastable form in Figs. 1(d) and 2(b), respectively, have been analyzed by using similar fitting protocols described above for the magnetic spectrum of the LP phase. This is to reduce the number of fitting parameters [28,30] (Tables S3 and S4). Minimally, three components with some degree of line broadening are employed for both large- $B_{h f}$ and small- $B_{h f}$ suites, yielding acceptably small residuals in the overall fit. This model accounts for additional sites not explicitly included in the deconvolution [38].

A representative fitting of $\mathrm{LuFe}_{2} \mathrm{O}_{4}-\mathrm{HP}$ is shown for the magnetic spectrum at $29 \mathrm{GPa}$ in Fig. 1(d). Three subcomponents with similar (linked) $I S=0.56 \mathrm{~mm} \mathrm{~s}^{-1}$ and relatively large- $\mathrm{B}_{\mathrm{hf}}$ values (31-36 $\mathrm{T}$, hatched yellow shading) account for resonances at high ( $x$-scale) velocities. Additionally three 
subspectra with small- $\mathrm{B}_{\mathrm{hf}}$ values (2-25 $\mathrm{T}$, shaded gray) and $I S=0.44 \mathrm{~mm} \mathrm{~s}^{-1}$ describe the asymmetric central region. A similar analysis is implemented for spectra in Fig. 2(b) of $\mathrm{LuFe}_{2} \mathrm{O}_{4}-\mathrm{HP}$ at ambient pressure, recovered from starting material compressed to $P \geqslant 12 \mathrm{GPa}$ [19]. Large- $\mathrm{B}_{\mathrm{hf}}$ components yield $I S=0.57 \mathrm{~mm} \mathrm{~s}^{-1}$, and small-B $\mathrm{B}_{\mathrm{hf}}$ components have $I S=0.68 \mathrm{~mm} \mathrm{~s}^{-1}$. The spectrum at $4 \mathrm{~K}$ in Fig. 2(b) was measured at the European Synchrotron Radiation Facility (ESRF, Grenoble, France) using synchrotron Mössbauer source methodology $[39,40]$. Yellow-shaded hatched and gray-shaded magnetic components evolve from large- and small-B $_{\text {hf }}$ subspectra at $300 \mathrm{~K}$, respectively, based on fitted IS values.

The valence separation plot in Fig. 2(c) compares IS values for fitted static subcomponents of the LP and HP phases of $\mathrm{LuFe}_{2} \mathrm{O}_{4}$ to those in $\mathrm{Fe}_{2} \mathrm{OBO}_{3}$ deemed to have mixed-integer valences [2,21]. The large valence separation in $\mathrm{Fe}_{2} \mathrm{OBO}_{3}$ with site-centered $\mathrm{CO}$ has $I S: \mathrm{Fe}^{2+} \geqslant 1 \mathrm{~mm} \mathrm{~s}^{-1}$ and $I S: \mathrm{Fe}^{3+} \leqslant 0.4 \mathrm{~mm} \mathrm{~s}^{-1}$ at ambient conditions [41]. For magnetic spectra of $\mathrm{LuFe}_{2} \mathrm{O}_{4}-\mathrm{HP}$ in Figs. 1(d) and 2(b), IS values in Fig. 2(c) correspond to suites of large- $\mathrm{B}_{\mathrm{hf}}$ (closed symbols) and small-B $\mathrm{B}_{\mathrm{hf}}$ subspectra (open symbols). The occurrence of both large- and small- $\mathrm{B}_{\mathrm{hf}}$ suites is additional evidence of some valence separation [27]. Figure 2(c) is analogous to bond-valence considerations in structural studies of mixed-valence compounds, including $\mathrm{Fe}_{2} \mathrm{OBO}_{3}$ and $\mathrm{LuFe}_{2} \mathrm{O}_{4}$ at ambient pressure [2,36].

At $300 \mathrm{~K}$, large- $\mathrm{B}_{\mathrm{hf}}$ and small- $\mathrm{B}_{\mathrm{hf}}$ components of $\mathrm{LuFe}_{2} \mathrm{O}_{4}$ $\mathrm{HP}$ do not have widely disparate $I S$ values anticipated for $\mathrm{Fe}^{2+}$ and $\mathrm{Fe}^{3+}$; see Fig. 2(c). The associated valences are more appropriately designated $\mathrm{Fe}^{(2+\Delta)+}$ and $\mathrm{Fe}^{(3-\Delta)+}$. The deconfinement coefficient $\Delta$ quantifies the time-averaged amount of minority-spin charge $\left(\Delta \times \mathrm{e}^{-}\right)$delocalized from $\mathrm{Fe}^{2+}$ to a neighboring $\mathrm{Fe}^{3+}$ site [42]. Its discernment in molecular complexes is known as valence detrapping, $0<$ $\Delta<0.5$, whereas $\Delta=0$ constitutes the valence trapped situation $[43,44]$. The consequential effect on measured IS values is that:

$$
\left(I S: \mathrm{Fe}^{(2+\Delta)+}\right)=(1-\Delta)\left(I S: \mathrm{Fe}^{2+}\right)+\Delta\left(I S: \mathrm{Fe}^{3+}\right) .
$$

A similar relation holds for $\left(I S: \mathrm{Fe}^{(3-\Delta)+}\right)$. Extrinsic shift contributions to $I S$ parameters are eliminated by taking differences of fitted values in Fig. 2(c), from which the electron deconfinement coefficient $\Delta$ may be obtained [45]:

$$
\begin{aligned}
& \left(I S: \mathrm{Fe}^{(2+\Delta)+}\right)-\left(I S: \mathrm{Fe}^{(3-\Delta)+}\right) \\
& \quad=\left\{\left(I S: \mathrm{Fe}^{2+}\right)-\left(I S: \mathrm{Fe}^{3+}\right)\right\}[1-2 \Delta] .
\end{aligned}
$$

The reference integer valence separation on the right-hand side of Eq. (2) stems from typical ionic $I S: \mathrm{Fe}^{2+}$ and $I S: \mathrm{Fe}^{3+}$ values of sixfold coordinated oxides, for example, $\mathrm{Fe}_{2} \mathrm{OBO}_{3}$ at ambient pressure in Fig. 2(c) [2,41].

Site-centered charge localization is when $\Delta=0$. Fully deconfined minority-spin electrons occurring at neighboring $\mathrm{Fe}^{2+}$ and $\mathrm{Fe}^{3+}$ sites with equal probability represent symmetric dimers $\Delta=0.5$. Partially delocalized minority-spin electrons, $0<\Delta<0.5$, have a higher probability of being at one of the neighboring sites in an asymmetric dimer due to a barrier to excursion, for example, from electron-lattice coupling $[46,47]$. If these mixed-valence cases manifest as long-range charge modulation, then these are site-centered $\mathrm{CO}(\Delta=0)$, bond-centered $\mathrm{CO}(\Delta=0.5)$, and an admixture (coexistence) of site- and bond-centered $\mathrm{CO}(0<\Delta<0.5)$. For symmetric dimers, statically fitted Mössbauer subspectra are indistinguishable and have the same $I S: \mathrm{Fe}^{2.5+}$ of $\sim 0.7 \mathrm{~mm} \mathrm{~s}^{-1}$ at $300 \mathrm{~K}[43,48]$. The $\mathrm{Fe}_{2} \mathrm{OBO}_{3}$ reference at pressure represents this case [21]; see Fig. 2(c). For asymmetric dimers, fitted IS and $\mathrm{B}_{\mathrm{hf}}$ parameters of subspectra representing $\mathrm{Fe}^{(2+\Delta)+}$ and $\mathrm{Fe}^{(3-\Delta)+}$ are distinguishable [44,47], as in $\mathrm{LuFe}_{2} \mathrm{O}_{4}-\mathrm{HP}$ in Figs. 2(b) and 2(c).

Note that the frequency of electron hopping or tunneling, $\mathrm{Fe}^{2+} \Leftrightarrow \mathrm{Fe}^{3+}$, must be considered with respect to the timesensing window of the probing technique. For MS, this "shutter speed" is $\sim 140 \mathrm{~ns}$, but it is much shorter for x-ray probes and determines what valences are discerned $[49,50]$.

\section{DISCUSSION}

Recovered $\mathrm{LuFe}_{2} \mathrm{O}_{4}-\mathrm{HP}$ at $300 \mathrm{~K}$ in Fig. 2(b) has $I S: \mathrm{Fe}^{(3-\Delta)+}=0.57 \mathrm{~mm} \mathrm{~s}^{-1}$ and $I S: \mathrm{Fe}^{(2+\Delta)+}=0.68 \mathrm{~mm} \mathrm{~s}^{-1}$ for large- $\mathrm{B}_{\mathrm{hf}}(27-32 \mathrm{~T})$ and small- $\mathrm{B}_{\mathrm{hf}}(4-22 \mathrm{~T})$ subspectra suites, respectively. Comparison with $\mathrm{Fe}_{2} \mathrm{OBO}_{3} \mathrm{LP}$ phase reference and using Eq. (2) yields $\Delta \sim 0.4$. Time-averaged valences for $\mathrm{Fe}$ constituents manifest as asymmetric dimers, denoted $\left[\mathrm{Fe}^{(2.4)+}: \mathrm{Fe}^{(2.6)+}\right]$. This emanates from comparatively fast minority-spin electron exchange, exceeding several megahertz [50]. An oxygen intermediary between neighboring Fe atoms facilitates electron exchange, $\mathrm{Fe}^{2+} \Leftrightarrow \mathrm{O} \Leftrightarrow \mathrm{Fe}^{3+}$ in Fig. 1(b), involving $\sigma$ bonding orbitals [42,51].

The increased valence (isomer shift) separation at $4 \mathrm{~K}(\Delta$ $\rightarrow$ 0.35), Fig. 2(c), suggests "freezing" of electron dynamics. Minority-spin carriers tend to dwell more at one of the Fe sites in a dimer as temperature is lowered. All $\mathrm{B}_{\mathrm{hf}}$ values increase as anticipated in an approach to saturation magnetization.

Figure 2(c) shows that, at in situ high pressures, IS values of large- and small- $\mathrm{B}_{\mathrm{hf}}$ components are less separated and are reversed in relative magnitudes compared to recovered $\mathrm{LuFe}_{2} \mathrm{O}_{4}$-HP. Nonetheless, large- and small- $\mathrm{B}_{\mathrm{hf}}$ spectral components are discerned, suggestive of valence separation similar to recovered $\mathrm{LuFe}_{2} \mathrm{O}_{4}-\mathrm{HP}$. This pressure evolution is therefore attributed to different pressure dependencies of ferrous- and ferric-type isomer shifts [21], analogous to their different temperature dependencies in Fig. 2(c). Integer valences $\mathrm{Fe}^{2+}$ and $\mathrm{Fe}^{3+}$ and site-centered $\mathrm{CO}$ can definitely be discounted, although it is less clear how asymmetric the dimers are at high pressures.

We also performed electronic structure calculations of $\mathrm{LuFe}_{2} \mathrm{O}_{4}$-HP by a full-potential linearized augmented plane wave method (WIEN2k) [52]. Cell parameters and two Lu and four Fe positions were taken from structural investigations of Damay et al. [19,38]. Oxygen positions were determined by structure relaxation and agreed closely with the HP recovered phase elucidated by Damay et al. [19]. The number of $k$ points in the Brillouin zone was 2000 (440 points in its irreducible part). The hybrid potential was the generalized gradient approximation with the Hubbard $U$ parameter GGA $+U$ form [53,54], with $U=4.61 \mathrm{eV}$ for $3 d$ iron states. Partial majority and minority electronic density of states (DOS) 

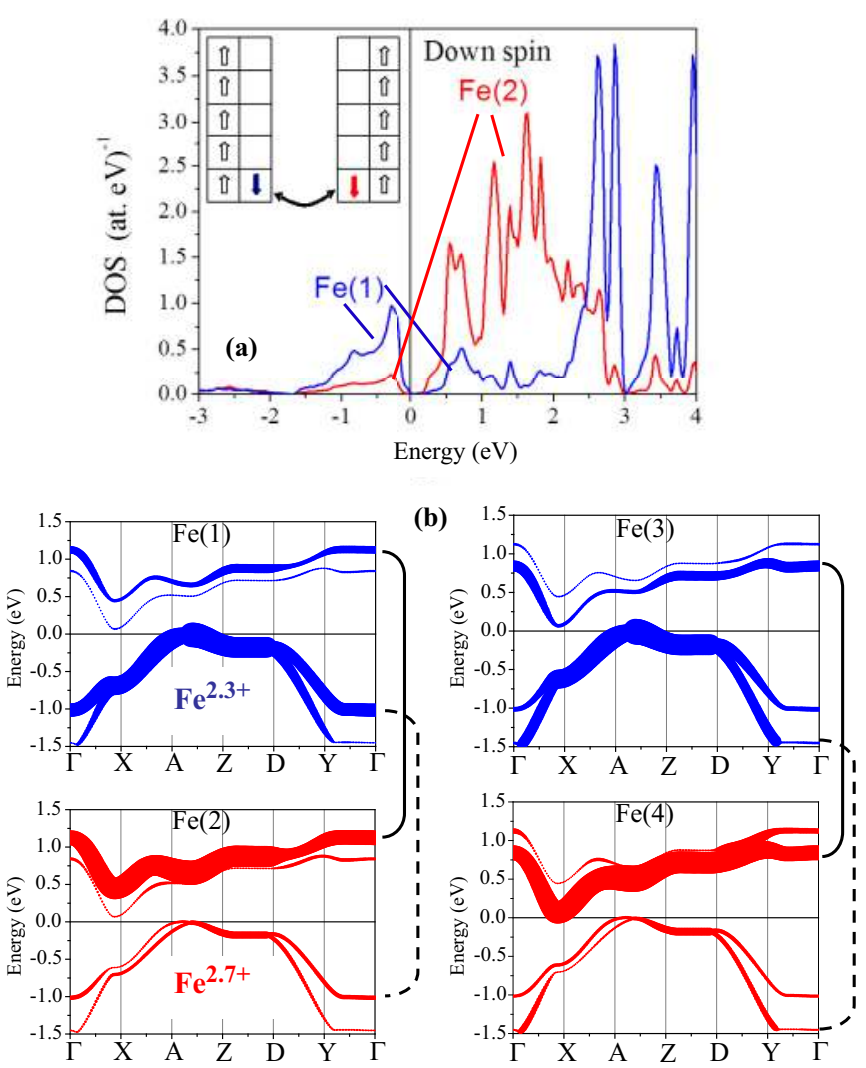

FIG. 3. (a) Partial density of minority-spin $3 d$ states at $\mathrm{Fe}(1)$ and $\mathrm{Fe}(2)$ sites of the HP phase. Inset depicts minority-spin electron hopping between neighboring sites. (b) Minority-spin band structure contribution from $3 d$ states near the Fermi level of each Fe site (weighting contribution to a subband represented by line thickness). The $\mathrm{Fe}(1)-\mathrm{Fe}(2)$ pair contributes higher weightings in upper subbands, while $\mathrm{Fe}(3)-\mathrm{Fe}(4)$ weightings dominate lower subbands. Filled minority-spin states occur at all Fe sites (dashed line highlights). A higher weight of filled states occurs at one site in both pairs, indicating asymmetric dimer formation.

profiles for the $3 d$ states are shown in Fig. 3(a) and in Fig. S2 of the Supplemental Material [28]. To smooth the DOS plots, we used a Gaussian broadening scheme with a width of $0.04 \mathrm{eV}$.

Calculations indicate that all $\mathrm{Fe}$ sites in $\mathrm{LuFe}_{2} \mathrm{O}_{4}-\mathrm{HP}$ are high spin; i.e., $3 d$ majority-spin states are filled. Based on the smoothing parameter used, the gap in minority spin states is deduced to be at least $\sim 100 \mathrm{meV}$, which is relatively close to that determined in electrical-transport measurements. Minorityspin $3 d$ states are mainly empty, but for all Fe sites, there is some fraction of minority-spin states below the Fermi level as shown for $\mathrm{Fe}(1)$ and $\mathrm{Fe}(2)$ sites; see Fig. 3(a). Integration of minority-spin DOS up to the Fermi energy gives the fractional number of electrons at these sites as 0.3 and 0.7 , implying asymmetric dimer formation $\left[\mathrm{Fe}^{(2.3)+}: \mathrm{Fe}^{(2.7)+}\right]$, compatible with our experimental findings. The electronic structure of both $\mathrm{Fe}(1)-\mathrm{Fe}(2)$ and $\mathrm{Fe}(3)-\mathrm{Fe}(4)$ pairs is essentially the same, although the four sites are structurally inequivalent.

To further verify dimer formation by $\mathrm{Fe}(1)-\mathrm{Fe}(2)$ and $\mathrm{Fe}(3)-\mathrm{Fe}(4)$ couples, we calculated the band structure in the vicinity of the Fermi level; see Fig. 3(b). Two flat subbands above and below the Fermi level, mainly from Fe $3 d$ states, are evident. Panels in Fig. 3(b) show contributions to minority-spin subbands from each Fe site (represented by subband line thickness). There are dominant upper subband contributions from $\mathrm{Fe}(1)$ and $\mathrm{Fe}(2)$ and dominant lower subband contributions from $\mathrm{Fe}(3)$ and $\mathrm{Fe}(4)$. This implies $3 d$ states of iron are strongly hybridized within $\mathrm{Fe}(1)-\mathrm{Fe}(2)$ and $\mathrm{Fe}(3)-\mathrm{Fe}(4)$ couples. Each pair contains a common $3 d$ minority-spin electron (filled states below the Fermi level), confined preferentially to one site in the pair. The sizable asymmetry of the dimers arises from the crystallographically inequivalent sites.

Asymmetric dimer formation in recovered $\mathrm{LuFe}_{2} \mathrm{O}_{4}-\mathrm{HP}$ deduced from IS values and electronic structure calculations in Figs. 2(c) and 3, respectively, consistently confirms the admixture of site-centered $(\Delta \sim 0)$ and bond-centered $(\Delta \sim 0.5)$ charge confinement. Recovered $\mathrm{LuFe}_{2} \mathrm{O}_{4}-\mathrm{HP}$ also evidences electron diffraction satellites in addition to Bragg reflections from the $P m$ parent unit cell [18,19]. If these Bragg satellites emanate from long-range charge modulation, then this is intermediate-state $\mathrm{CO}$, because site-centered $\mathrm{CO}$ is precluded by Fig. 2(c). An asymmetric dimer phase is predicted to render ferroelectricity because it breaks spatial inversion symmetry $[3,6]$.

In $\mathrm{LuFe}_{2} \mathrm{O}_{4}-\mathrm{HP}$, ferromagnetic spins occur in the $a$ direction. There are also alternating $\sim 3.00 \AA$ and $\sim 2.80 \AA$ Fe-Fe distances, reminiscent of a Peierls distortion [19]; see Fig. 2(d). Along the $b$ direction Fe-Fe distances are longer (3.29 $\AA$ ) for neighboring antiferromagnetic spins [19]. The $a$ direction has optimum Fe-O-Fe orbital overlap for Zener double-exchange involving ferromagnetic spins; see Figs. 1(b) and 2(d) [11]. It is the geometrically and energetically favored pathway for $\sigma$-bonded minority-spin electron deconfinement and consequent dimer formation $[55,56]$.

Corroborative temperature-dependent resistivity-pressure data in Fig. 2(e) show that the pressure dependence of $\rho$ in $\mathrm{LuFe}_{2} \mathrm{O}_{4}-\mathrm{HP}$ is much lower than in the LP phase. Thus, charge carrier characteristics change at the LP $\rightarrow \mathrm{HP}$ structural transition. The spectral analysis in Figs. 2(b) and 2(c) suggests minority-spin electron hopping $\mathrm{Fe}^{2+} \Leftrightarrow \mathrm{Fe}^{3+}$ involving nearest neighbors in $\mathrm{LuFe}_{2} \mathrm{O}_{4}-\mathrm{HP}$, as shown in Fig. 2(d). This hopping is prone to a Jahn-Teller induced lattice polarization barrier $[4,13]$. Consequently, $\mathrm{LuFe}_{2} \mathrm{O}_{4}-\mathrm{HP}$ resistivity data were analyzed as thermally activated small polaron motion $\rho / T \propto \exp \left(W_{H} / k_{B} T\right)$, valid for $T>\theta_{D} / 2$, where $W_{H}$ is the thermal activation barrier for hopping, and $\theta_{D} / 2$ is half the Debye temperature $150-200 \mathrm{~K}$ [57]. Best fits to linearized data from 180 to $320 \mathrm{~K}$ [Fig. 2(e)] yield $W_{H}=230 \mathrm{meV}$ at $\sim 25 \mathrm{GPa}$ and $\sim 270 \mathrm{meV}$ in decompressed (recovered) $\mathrm{LuFe}_{2} \mathrm{O}_{4}-\mathrm{HP}$ at $\sim 1 \mathrm{GPa}$.

\section{CONCLUDING REMARKS}

$\left[\mathrm{Fe}_{2} \mathrm{O}_{4}\right]_{\infty}$ bilayer substructures persist in $\mathrm{LuFe}_{2} \mathrm{O}_{4}-\mathrm{HP}$, and a rectangular network of Fe atoms occurs [19]; see Fig. 1(b). Nearest-neighbor $\mathrm{Fe}-\mathrm{Fe}$ interatomic distances both within and between sheets of a bilayer are smaller than respective distances in the triangular networked LP phase. Evidently, sitecentered $\mathrm{CO}$ is incompatible with this rectangular network and its reduced interatomic spacings. This triggers back-and-forth minority-spin electron migration between neighboring Fe sites 
and the formation of asymmetric dimers $\left[\mathrm{Fe}^{(2.4)+}: \mathrm{Fe}^{(2.6)+}\right]$. This is a lower repulsive energy situation than in statically configured $\mathrm{Fe}^{3+}$ and $\mathrm{Fe}^{2+}$ valences of site-centered $\mathrm{CO}$ [47]. A superstructure of asymmetric dimers rationalizes the additional charge modulation (Bragg satellites) discerned in electron diffraction experiments of recovered HP phase at $300 \mathrm{~K}$. Asymmetric dimers in the metastable HP magnetic phase of $\mathrm{LuFe}_{2} \mathrm{O}_{4}$ potentially manifest as intermediate-state $\mathrm{CO}$ at ambient conditions. This yields the requisite inversionsymmetry breaking for CO electronically-invoked ferroelectricity in the metastable HP polymorph.

\section{ACKNOWLEDGMENTS}

We acknowledge receipt of the samples from Christine Martin (Laboratoire de Cristallographie et Sciences des Matériaux, Caen-France) and extensive discussions with Françoise Damay (Saclay-France) and Jerome Rouquette (Montpellier-France). Rudolph Rüffer, Aleksandr Chumakov, and support staff provided professional assistance for the synchrotron Mössbauer source measurements at cryogenic temperatures, at the ID-18 beamline of the ESRF. Funding for this research was provided by the National Research Foundation of South Africa under Grant No. 87808.
[1] This localization in strongly correlated electron systems is a mechanism to minimize the repulsive energy associated with onsite Hubbard $U$ and longer-range intersite $V$ interactions. This is at the expense of the gain in kinetic energy from delocalization signified by the hopping integral $t$.

[2] M. Angst, P. Khalifah, R. P. Hermann, H. J. Xiang, M.-H. Whangbo, V. Varadarajan, J. W. Brill, B. C. Sales, and D. Mandrus, Phys. Rev. Lett. 99, 086403 (2007).

[3] D. V. Efremov, J. van den Brink, and D. I. Khomskii, Nat. Mater. 3, 853 (2004).

[4] D. I. Khomskii, Transition Metal Compounds (Cambridge University Press, Cambridge, UK, 2014).

[5] G. Giovannetti, S. Kumar, D. Khomskii, S. Picozzi, and J. van den Brink, Phys. Rev. Lett. 103, 156401 (2009).

[6] J. van den Brink and D. I. Khomskii, J. Phys.: Condens. Matter 20, 434217 (2008).

[7] H. Seo, C. Hotta, and H. Fukuyama, Chem. Rev. 104, 5005 (2004).

[8] G. Giovannetti, S. Kumar, J. van den Brink, and S. Picozzi, Phys. Rev. Lett. 103, 037601 (2009).

[9] R. Comin, R. Sutarto, F. He, E. H. d. S. Neto, L. Chauviere, A. Fraño, R. Liang, W. N. Hardy, D. A. Bonn, Y. Yoshida et al., Nat. Mater. 14, 796 (2015)

[10] E. da Silva Neto, P. Aynajian, A. Frano, R. Comin, E. Schierle, E. Weschke, A. Gyenis, J. Wen, J. Schneeloch, Z. Xu et al., Science 343, 393 (2014).

[11] A. Daoud-Aladine, J. Rodríguez-Carvajal, L. Pinsard-Gaudart, M. T. Fernández-Díaz, and A. Revcolevschi, Phys. Rev. Lett. 89, 097205 (2002).

[12] L. Wu, R. F. Klie, Y. Zhu, and C. Jooss, Phys. Rev. B 76, 174210 (2007).

[13] C. Jooss, L. Wu, T. Beetz, R. F. Klie, M. Beleggia, M. A. Schofield, S. Schramm, J. Hoffmann, and Y. Zhu, Proc. Nat. Acad. Sci. 104, 13597 (2007).

[14] N. Ikeda, J. Phys.: Condens Matter. 20, 434218 (2008).

[15] A. Nagano, M. Naka, J. Nasu, and S. Ishihara, Phys. Rev. Lett. 99, 217202 (2007).

[16] M. Angst, Phys. Stat. Sol. RRL 7, 375 (2013).

[17] I. K. Yang, J. Kim, S. H. Lee, S.-W. Cheong, and Y. H. Jeong, Appl. Phys. Lett. 106, 152902 (2015).

[18] J. Rouquette, J. Haines, A. Al-Zein, P. Papet, F. Damay, J. Bourgeois, T. Hammouda, F. Doré, A. Maignan, M. Hervieu et al., Phys. Rev. Lett. 105, 237203 (2010).

[19] F. Damay, M. Poienar, M. Hervieu, A. Guesdon, J. Bourgeois, T. Hansen, E. Elkaïm, J. Haines, P. Hermet, L. Konczewicz et al., Phys. Rev. B 91, 214111 (2015).
[20] K. Momma and F. Izumi, J. Appl. Crystall. 44, 1272 (2011).

[21] G. R. Hearne, W. N. Sibanda, E. Carleschi, V. Pischedda, and J. P. Attfield, Phys. Rev. B 86, 195134 (2012).

[22] G. Diguet, G. R. Hearne, W. N. Sibanda, E. Carleschi, P. Musyimi, V. Pischedda, and J. P. Attfield, Phys. Rev. B 89, 035132 (2014).

[23] J. Bourgeois, M. Hervieu, M. Poienar, A. M. Abakumov, E. Elkaïm, M. T. Sougrati, F. Porcher, F. Damay, J. Rouquette, G. V. Tendeloo et al., Phys. Rev. B, 85, 064102 (2012).

[24] M. Hervieu, A. Guesdon, J. Bourgeois, E. Elkaïm, M. Poienar, F. Damay, J. Rouquette, A. Maignan, and C. Martin, Nat. Mat. 13, 74 (2014).

[25] Z. Klencsár, MossWinn 4.0Pre, http://www.mosswinn.com/.

[26] The hyperfine interaction parameters including Fe site abundances are derived from fitting the spectral envelope with Lorentzian subcomponents. The isomer (chemical) shift $I S$ is proportional to the $s$-electron density at the Fe nucleus, and it is influenced by $d$-electron shielding. The quadrupole doubletsplitting or line shift, $Q S$, is proportional to the deviation from cubic symmetry of both the surrounding electron configuration and nearest-neighbor atoms. Both $I S$ and $Q S$ have distinct "fingerprint" values for $\mathrm{Fe}^{2+}\left(3 d^{6}\right)$ and $\mathrm{Fe}^{3+}\left(d^{5}\right)$ ions. In complex spectra, with appreciable overlap of subcomponents, mainly $I S$, and to a lesser extent $\mathrm{B}_{\mathrm{hf}}$ values (from magnetically split hyperfine structure), of fitted subspectra are obtained with good reliability, to establish valences of Fe species.

[27] The magnetic hyperfine field is a result of the nuclear Zeeman splitting of ${ }^{57} \mathrm{Fe}$ nuclear levels due to an internal magnetic field at the nucleus. The primary contribution to this internal field, $\mathrm{B}_{\mathrm{hf}}$, is from the contact term. This is the effect of $d$-electron spin polarization on the $s$-electrons. Other contributions to $\mathrm{B}_{\mathrm{hf}}$ are from comparatively small dipolar and orbital terms. In ferric iron, the orbital and dipolar terms are negligibly small. The orbital term in ferrous iron may be appreciable and of opposite sign to the contact term. Hence, $\mathrm{B}_{\mathrm{hf}}$ in ferrous compounds tends to be much smaller than in ferric compounds. At well below the magnetic ordering temperatures, $\mathrm{B}_{\mathrm{hf}}$ in ferric oxides is typically clustered around $\sim 50 \mathrm{~T}$, whereas in ferrous compounds, it may range from near zero to $44 \mathrm{~T}$, depending on the orbital configuration.

[28] See Supplemental Material at http://link.aps.org/supplemental/ 10.1103/PhysRevB.93.105101 for further description of experiments, tables of parameters from Mössbauer analyses, and supplementary information and plots for both resistivity pressure study and electronic structure calculations. 
[29] T. Maruyama, Y. Murakami, D. Shindo, N. Abe, and T. Arima, Phys. Rev. B 86, 054202 (2012).

[30] A first-order approximation to the Hamiltonian with mixed magnetic and quadrupole interactions is used to obtain the spectral profile (line intensities and positions) of the large- $\mathrm{B}_{\mathrm{hf}}$ subspectra. The full Hamiltonian is used to profile the small- $\mathrm{B}_{\mathrm{hf}}$ subcomponents, which is appropriate when the quadrupole interaction is the same order of magnitude as the magnetic hyperfine interaction. The $I S$ values of the large- $\mathrm{B}_{\mathrm{hf}}$ components are linked to be the same and similarly for the suite of small- $B_{\mathrm{hf}}$ subspectra.

[31] This broadened sextet is ascribed to frustrated (disordered) $\mathrm{Fe}^{2+}$ spins (fluctuating or a distribution of $\mathrm{B}_{\mathrm{hf}}$ values) anticipated to occur in the triangular network. Longer-range magnetic exchange interactions within the bilayers give rise to the spinaligned (ordered) components.

[32] S. Nakamura, H. Kito, and M. Tanaka, J. Alloys Comp. 275-277, 574 (1998).

[33] B. K. Bang, S. B. Kim, S.-W. Cheong, and C. S. Kim, Phys. Status Solidi (B) 244, 4566 (2007).

[34] A. M. Mulders, M. Bartkowiak, J. R. Hester, E. Pomjakushina, and K. Conder, Phys. Rev. B 84, 140403 (2011).

[35] K.-T. Ko, H.-J. Noh, J.-Y. Kim, B.-G. Park, J.-H. Park, A. Tanaka, S. B. Kim, C. L. Zhang, and S.-W. Cheong, Phys. Rev. Lett. 103, 207202 (2009).

[36] J. de Groot, T. Mueller, R. A. Rosenberg, D. J. Keavney, Z. Islam, J.-W. Kim, and M. Angst, Phys. Rev. Lett. 108, 187601 (2012).

[37] S. M. Gaw, H. J. Lewtas, D. F. McMorrow, J. Kulda, R. A. Ewings, T. G. Perring, R. A. McKinnon, G. Balakrishnan, D. Prabhakaran, and A. T. Boothroyd, Phys. Rev. B 91, 035103 (2015).

[38] Four Fe positions in the $P m$ unit cell have been obtained in the refinement of structural data by Damay et al. [19]. They acknowledge that the "true" oxygen network is more complex, with a likely distribution of $\mathrm{Fe}-\mathrm{O}$ distances on the different $\mathrm{Fe}$ sites.

[39] V. Potapkin, A. I. Chumakov, G. V. Smirnov, J.-P. Celse, R. Rüffer, C. McCammon, and L. Dubrovinsky, J. Synchrotron Rad. 19, 559 (2012).

[40] This source has a $\sim 25 \%$ broader line width than the "point" source used in our in-house experiments. Nevertheless, the tightly focused high photon flux and exceedingly low nonresonant background are efficacious for investigating microscopic samples at liquid helium temperatures.

[41] A. P. Douvalis, V. Papaefthymiou, A. Moukarika, T. Bakas, and G. Kallias, J. Phys.: Condens. Matter 12, 177 (2000).

[42] In octahedral coordination, this minority-spin electron would reside in a $t_{2 \mathrm{~g}}$ orbital. It is more appropriate to refer to these as being in $\sigma$-bonding orbitals in the very low crystal symmetry and local symmetry at iron sites in the HP structure of $\mathrm{LuFe}_{2} \mathrm{O}_{4}$.

[43] V. Papaefthymiou, J.-J. Girerd, I. Moura, J. J. G. Moura, and E. Münck, J. Amer. Chem. Soc. 109, 4703 (1987).
[44] X.-Q. Ding, E. Bill, A. X. Trautwein, H. Winkler, A. Kostikas, V. Papaefthymiou, A. Simoupoulos, P. Beardwood, and J. F. Gibson, J. Chem. Phys. 99, 6421 (1993).

[45] We use the isomer shift separation in Eq. (2) rather than the absolute value in Eq. (1) to obtain the deconfinement coefficient $\Delta$. This eliminates the effect of any extrinsic shifts that may occur in the centroid of a fitted subspectrum, for example, from instrumental effects or data reduction and analysis procedures.

[46] V. Coropceanu, V. Schünemann, C. Ober, M. Gerdan, A. X. Trautwein, J. Köhler, and R. W. Saalfrank, Inorg. Chim. Acta 300-302, 875 (2000).

[47] P. Karen, P. M. Woodward, J. Lindén, T. Vogt, A. Studer, and P. Fischer, Phys. Rev. B 64, 214405 (2001).

[48] X.-Q. Ding, E. L. Borninaar, E. Bill, H. Winkler, A. X. Trautwein, S. Drüeke, P. Chaudhuri, and K. Wieghardt, J. Chem. Phys. 92, 178 (1990).

[49] P. Gütlich, E. Bill, and A. X. Trautwein, Mössbauer Spectroscopy and Transition Metal Chemistry: Fundamentals and Applications (Springer, Berlin, 2011).

[50] For the ${ }^{57} \mathrm{Fe}$ MS probe, the "shutter speed" is the lifetime of the nuclear excited state $(\tau \sim 140 \mathrm{~ns})$. The MS technique would register a nonintegral valence if the electron-hopping frequency $\omega_{E}$ far exceeded $1 / \tau \sim 7 \mathrm{MHz}$. If the electron exchange process is slow, $\omega_{E} \ll 1 / \tau$, then discrete valences (large valence separation and a corresponding large difference in $I S$ values) will be discerned. Note that this may be different to what would be revealed by other fast probes of Fe valence or charge modulation, like x-ray absorption spectroscopies or diffraction techniques. These have much shorter sensing times (core-hole lifetime $\tau \sim 10^{-17} \mathrm{~s}$ ) than Mössbauer spectroscopy. In these fast snapshots of electron-exchange that range from several to a few hundred megahertz (comparatively slow dynamical processes), separate $\mathrm{Fe}^{2+}$ and $\mathrm{Fe}^{3+}$ valences would still be discerned by these techniques.

[51] The $d$-electron from site $i$ is transferred to a neighboring site $j$ via an intermediate oxygen. The hopping occurs as a two-step process : $d_{i}{ }^{n} p^{6} d_{j}{ }^{n} \rightarrow d_{i}{ }^{n} p^{5} d_{j}{ }^{n+1} \rightarrow d_{i}{ }^{n-1} p^{6} d_{j}{ }^{n+1}$.

[52] P. Blaha, K. Schwarz, P. Sorantin, and S. B. Trickey, Comput. Phys. Commun. 59, 399 (1990).

[53] V. I. Anisimov, I. V. Solovyev, M. A. Korotin, M. T. Czyzyk, and G. A. Sawatzky, Phys. Rev. B 48, 16929 (1993).

[54] J. P. Perdew, K. Burke, and M. Ernzerhof, Phys. Rev. Lett. 77, 3865 (1996).

[55] J.-J. Girerd, V. Papaefthymiou, K. K. Surerus, and E. Münck, Pure Appl. Chem. 61, 805 (1989).

[56] The most energetically favorable process involving minorityspin electron delocalization in a background of aligned atomic spins dictates that the orientation of atomic spins of Fe neighbors should be ferromagnetic. In this case, an energy penalty involving the Hund's rule stabilization energy, $J_{H} \sim 1 \mathrm{eV}$, is not incurred in the electron transfer process.

[57] M. Ziese and C. Srinitiwarawong, Phys. Rev. B 58, 11519 (1998). 